\title{
Percepción de la delincuencia y miedo al crimen con relación a los medios de comunicación en Costa Rica
}

\author{
Stephanie Fernández-Brizuela \\ Universidad de Iberoamérica
}

\begin{abstract}
Resumen
La percepción de la delincuencia en la población se ve afectada en alguna medida por los medios de comunicación. Esta investigación cuantitativa, correlacional y transversal, analizó la relación entre la percepción de la delincuencia y el consumo de medios de comunicación en una muestra de costarricenses mayores de edad utilizando la Escala de Miedo al Crimen y la Escala de Transporte Narrativo. Los datos se recabaron de manera virtual durante el mes de octubre del 2020 con la participación de 157 costarricenses que consumían noticias delictivas a través de algún medio de comunicación. Los resultados evidenciaron que no existe una relación entre el miedo y el consumo semanal de noticias ni tampoco una relación entre la edad y la percepción del delito; sin embargo, se halló que las mujeres y personas de un estrato social bajo perciben de forma más negativa el fenómeno de la delincuencia a partir del miedo al crimen, el cual, a su vez, se encuentra significativamente relacionado con el transporte narrativo.
\end{abstract}

Palabras clave: Delincuencia, miedo, crimen, medios de comunicación.

\begin{abstract}
The perception of delinquency in the general population is affected by the media somehow. This quantitative, correlational and transversal research analyzed the relation between the perception of crime and the consumption of media in Costa Rican adults using the Scale of Fear of Crime and the Scale of Narrative Transportation. The data was collected virtually during October 2020 with the participation of 157 Costa Ricans who consumed crime news through media. Results showed that there is no relation between fear and weekly news consumption or a relation between age and the perception of crime. Instead, it was found that women and individuals from lower social classes perceived the crime phenomenon in a more negative way from fear of crime, which, in turn, is significantly related to narrative transport.
\end{abstract}

Key words: Delinquency, fear, crime, media. 


\section{Introducción}

La delincuencia ha sido un constructo altamente estudiado, donde los medios de comunicación han tomado un papel protagónico en la percepción social de las personas (Soto, 2005). Los medios de comunicación influencian de una manera importante las definiciones, imágenes, impresiones y sensaciones; así como expresan valores y juicios normativos. Esto aplica de igual forma para los noticiarios, los cuales son consumidos a través de múltiples procesos sensoriales y cognitivos subyacentes (Carmona, 2002; Villalba, 2017).

La percepción respecto a la delincuencia está en gran medida influenciada tanto por su propia experiencia como víctima, así como la de personas cercanas, constituyendo los noticiarios de igual forma otro elemento de socialización en la conformación de la experiencia (Soto, 2005). De esta forma, las anteriores formas de exposición a conductas delictivas van forjando un grado de sospecha personal hacia supuestos asaltantes o delincuentes (García-Castro y Barrantes-Umaña, 2016).

García-Castro y Pérez-Sánchez (2018) señalan que la percepción del crimen suele ser mayor de lo que realmente es. En su estudio encontraron que la brecha entre la probabilidad percibida de ser víctima de un delito violento sobre la real es 2,1 veces mayor en el caso de delitos contra la propiedad, 16,7 para violencia física y 11 veces para violencia emocional.

Téllez (2015) ha indicado que existen distintos factores estructurales que contribuirían a la percepción de inseguridad, como el desempleo, la violencia general -delincuencia-, calidad en cuanto a la salud y educación, así como la capacidad de control formal e informal dada por el control social y los medios de comunicación. Tal es el caso del Triángulo Norte de Centroamérica (Guatemala, El Salvador y Honduras) y el Norte de México, con la violencia de posguerra, el narcotráfico y la violencia política; o Nicaragua, donde parece ser que la violencia es común desde hace mucho tiempo (Huhn, 2012).

En Costa Rica, uno de los hallazgos más significativos en torno a una investigación histórica- comparativa de la criminalidad, se sustenta en el análisis de los "discursos a la nación" de los presidentes de Costa Rica desde finales de los años 40 hasta el siglo XX. Así, a lo largo de los años 50 hasta los 90 se evidenció que el Estado poseía “conocimientos válidos" para entender el fenómeno de la criminalidad, constituyéndose como un problema de interés general, de manera que en los 50 se demarcó como un problema de la sociedad, la juventud y la educación; concepción que se modificó para hacer el problema uno moral, social y de consumo de drogas hasta que se convirtió en un problema de extranjeros socialmente construido en los años 80 y en el presente un problema generalmente ubicado fuera de la sociedad, pero atacándola. En la criminalidad de los años 80 y 90, se venían dando aproximaciones en torno al delito como es entendido en la actualidad, dado que en dicho período fue cuando el fenómeno empezó a ser explicado a partir de estadísticas criminales, medios de comunicación, el discurso cotidiano y los esfuerzos insuficientes del Estado por combatir la delincuencia. Además, en los años 90, el expresidente Calderón enfatizó el problema de la inseguridad ciudadana como uno de los más grandes del país, de modo que prometió, que en caso de ser electo reformaría las leyes y haría del sistema penitenciario un verdadero instrumento de rehabilitación para las personas privadas de libertad. Igualmente, en el 
ideario costarricense de 1993, el politólogo Francisco Barahona ligó la creciente violencia en el país con la televisión como una mala influencia, pero sugiriendo que la criminalidad era sobre todo una señal de que se necesitaba un nuevo modelo de desarrollo en el país (Huhn, 2012).

Con respecto a lo anterior, se destaca el estudio de García-Castro (2012) cuyo objetivo se centró en conocer qué relación tiene consumir noticias televisivas/televisión y la percepción de criminalidad, como su relación con aspectos socio demográficos e ideológicos y el impacto diferenciado en las clases populares y en la clase alta del país. Así, a partir de una muestra de 151 personas, los hallazgos demostraron que el consumo televisivo está relacionado con el miedo difuso y el riesgo percibido, de manera que son los individuos pertenecientes a la clase social baja quienes más ven telenoticieros. A su vez, el visionado de telenoticieros y la percepción de criminalidad incidieron en las creencias sobre justificación del sistema. Además, el miedo difuso repercutió en el apoyo militar estadounidense en Costa Rica y la percepción de criminalidad en el prejuicio hacia los inmigrantes (nicaragüenses). También, se resaltó que la clase social incidió en el miedo difuso y los sujetos pertenecientes a la clase social baja fueron quienes más miedo reportaron.

De todas formas, la evidencia de la relación del consumo de noticias televisivas y la percepción del crimen no resulta un hecho aislado. Callanan (2012), realizó un estudio con 3712 californianos cuyo objetivo fue examinar el impacto de los medios (periódicos, noticieros locales, programas de realidad de crimen televisivo y dramas criminales televisivos) relacionados con la delincuencia, en las percepciones de los individuos sobre el miedo al delito, luego comparar estos efectos entre los blancos, latinos y grupos afroamericanos. Dentro de los resultados, se hallaron diferencias en el impacto de los medios, relacionados con la delincuencia por diferentes medios de comunicación y grupos étnicos, de manera que la televisión fue el que más percepciones de riesgo y miedo al crimen causó para todos los grupos, mientras que el consumo de periódicos y de drama criminal fueron más variables.

En Chile, mientras tanto, parece no haber una asociación estadísticamente significativa entre exposición medial a noticias policiales en la televisión y el temor a la delincuencia; no obstante, parece que el temor al crimen es explicado a partir de que los más temerosos frente al crimen son quienes más prestan atención a noticias de carácter delictivo (precisamente por su alta preocupación ante el tema), lo cual es argumentado a partir de la Teoría de Usos y Gratificaciones, la cual indica que la información está mediada por las necesidades personales que prescinden de la dirección de la atención hacia ciertos contenidos, implicando la realización de ciertos usos para obtener ciertas gratificaciones que permitan satisfacer las carencias iniciales (Álvarez-Gálvez, 2012; Browne \& Tomicic, 2011). Se debe tomar en cuenta que, en palabras de Focás (2018), en el contexto chileno, es menos común que las noticias sean sensacionalistas, de modo que se suele proteger la identidad de la víctima; caso contrario a lo que sucede en Perú donde suelen poner la fotografía e inclusive el nombre completo de la persona. 
Más recientemente, Vergara (2016) realizó un análisis de discurso sobre la criminalidad en textos informativos televisivos y su relación con una percepción "desmedida" respecto de la delincuencia por parte de la mayoría de la población en Costa Rica. Se aplicaron conceptos de la lingüística, el análisis del discurso y el análisis crítico del discurso, además de teorías sociales y psicológicas con 6 noticias televisivas: 4 trasmitidas por Teletica y 2 por Repretel de sus canales 7 (Telenoticias) y 6 (Noticias Repretel) respectivamente. Los resultados revelaron que los homicidios ocupan un nivel superior dentro del discurso. Además, se dio la identificación de algunos actores permanentes (víctimas, victimarios, posibles victimarios, posibles víctimas, medios de comunicación y órganos de control social) donde se concibe a la víctima como inocente, pasiva e identificada, mientras que los victimarios se caracterizan por ser violentos, incontrolables, activos y desconocidos. Así, los medios de comunicación se presentan a sí mismos como expertos, explicando y categorizando los eventos, así como emitiendo juicios de valor sobre los actores. Finalmente, los órganos de control social se muestran en el cumplimiento de una parte de su trabajo como expertos, así como en una condición precaria y nunca se valora sus actos, de manera que no se emiten juicios de valor positivos respecto a sus acciones, porque son su obligación. Dentro de otros hallazgos, se expuso el discurso sobre la criminalidad manifestada en los textos informativostelevisivos a partir de las causas y consecuencias del crimen; las cargas afectivas fuertes como la muerte, violencia, peligro, personalización, victimización y criminalización; y la "realidad" social de Costa Rica, o sea, la representación de un contexto social. En síntesis, dicho estudio permitió evidenciar que las características del discurso tienen la capacidad de producir miedo en una parte de la población y, por ende, juegan un papel importante -junto a discursos emitidos por otras fuentes- en la sensación de inseguridad en Costa Rica.

Sumado a lo anterior, García-Castro y Pérez-Sánchez (2018) investigaron la asociación entre el consumo general de televisión, determinadas noticias de televisión y el miedo al delito, tomando en consideración la teoría del Modelo del Transporte Narrativo y la Teoría de la Justificación del Sistema.

Por un lado, el Modelo de Transporte Narrativo contempla la sensación mediada por la atención, implicación emocional y capacidad cognitiva para entrar en un mundo evocado por la narrativa, esto debido a la empatía por los personajes de la historia y la imaginación de la trama, donde las experiencias emocionales negativas pueden ocurrir cuando los consumidores son transportados en narrativas desagradables, como por ejemplo en las noticias en torno al crimen (García-Castro y Pérez-Sánchez, 2018; Green, 2004; Van Laer et al., 2014).

Por el otro lado, la Teoría de Justificación de Sistema se basa en "una construcción cognoscitiva, que se fundamenta desde la motivación del individuo a legitimar el status quo percibiéndolo como necesario, natural, justo e inevitable" (Jaume y Etchezahar, 2013, párr. 12). 
De lo anterior que las personas tiendan a realizar evaluaciones de las instituciones a partir de la necesidad de mantener un sentido de certidumbre y estabilidad, sentir seguridad y confianza, y de afiliarse a otros individuos que forman parte de los sistemas sociales; así como evadir el miedo a la delincuencia en torno de la ilusión de control, defendiendo el status quo para minimizar la incertidumbre y tener más control en sus vidas con una reducción de la ansiedad, la incertidumbre y el miedo (García-Castro y Pérez-Sánchez, 2018; Jaume y Etchezahar, 2013; Jost, Banaji y Nosek, 2004; Jost y Hunyady, 2005; Jost, Ledgerwood y Hardin, 2008).

Así, García-Castro y Pérez-Sánchez (2018) condujeron su estudio a partir de una muestra de 516 estudiantes costarricenses. Para el análisis, se consideraron el transporte narrativo y la justificación del sistema como variables predictoras psicológicas; además, sexo, nivel de delincuencia en el área de residencia y clase social como covariables sociales. Utilizaron la Escala de Miedo al Crimen, la Escala Narrativa de Transporte y la Escala de Justificación del Sistema, en donde se encontró una correlación entre miedo al crimen con personas que experimentaron más transporte narrativo, menos justificación del sistema, mujeres y clase social baja.

Los hechos sugieren que el consumo televisivo juega un papel importante en la percepción del miedo hacia el delito, de forma que las personas de estrato social bajo son las que reportan dicho fenómeno, pues existe una correlación entre la clase social baja y la obtención de información a través de la televisión; caso contrario a lo que sucede en los estratos medio y alto, donde el medio de comunicación predilecto para informarse es el periódico (Fonseca y Sandoval, 2005).

Otro aspecto a considerar es la inseguridad en América Latina, la cual se podría decir que es producto del vacío que han dejado las políticas de bienestar social, acompañada del aumento en la oferta de los medios de comunicación sobre noticias en torno a la criminalidad, cuyo discurso ideológico no necesariamente corresponde a la experiencia real, pero que aún bajo la concepción ficticia, generan consecuencias tangibles, tales como el aumento del cuerpo policial (sobre todo en vía pública y en lugares donde concurren las clases populares), reformas legislativas y costes políticos elevados (Huhn, 2012).

Así, se podría inferir que la percepción del delito se encuentra mediada por el contacto directo con el crimen, la experiencia trasmitida por personas allegadas y la información suministrada por los medios de comunicación; de modo que dicha tríada da lugar -a su vez- al miedo al crimen, donde la inseguridad y desigualdad social también parecen tener un papel protagónico. De ahí que los países industrializados suelan presentar cifras delictivas inferiores a los que se encuentran en vías de desarrollo. 
En Costa Rica, por ejemplo, entre los años 1994 y 1997 se registró un aumento en los delitos contra la propiedad, así como los cometidos contra la vida humana en lo relativo a la agresión con arma; mientras que la tasa de homicidios dolosos se mantuvo relativamente estable, donde 3 de cada 4 casos tenían como imputado a una persona conocida de la víctima (Sáenz, 2003). Más aún, las cifras de menor antigüedad parecen ser aún más alarmantes, pues desde enero del 2015 hasta junio del 2018 se perpetuaron 202191 delitos en el país, donde fue San José la provincia que recabó más crímenes con un total de 75222 eventos, seguida por Alajuela con 32542, Puntarenas con 25306, Heredia con 19291, Limón con 18242, Guanacaste con 16782 y Cartago con 14806 (Poder Judicial de Costa Rica, 2018).

Por tanto, a propósito de lo anterior, con el aumento abrupto de la delincuencia y la incertidumbre de determinar cuál es el medio de comunicación predilecto para los estratos sociales en la actualidad, la presente investigación tiene como objetivo general analizar la relación entre la percepción de la delincuencia y el consumo de medios de comunicación en una muestra de costarricenses mayores de edad. Adicionalmente, esta pretende analizar la relación entre el sentimiento de miedo al crimen y el transporte narrativo en el consumo de noticias, analizar la relación entre la percepción de la delincuencia y el estatus socioeconómico, identificar el género que percibe más negativamente el fenómeno de la delincuencia y describir la percepción de la delincuencia en función de la edad; planteando que, (H1) a menor clase social, se encontraría una mayor percepción negativa de la delincuencia, $(\mathrm{H} 2)$ el sexo femenino tiene una percepción de la delincuencia más negativa que el sexo masculino, (H3) el medio de comunicación por el que se consumen más noticias delictivas son las redes sociales y (H4) a mayor edad, mayor percepción negativa de la delincuencia.

\section{Método}

\section{Participantes}

El estudio contó con la participación de 157 costarricenses mayores de edad, los cuales tenían acceso a algún medio de comunicación y al consumo de noticias delictivas. La evaluación de los participantes fue voluntaria, sin ninguna compensación por su contribución a la investigación, lo cual se denotó con la aceptación del consentimiento informado donde se expuso el propósito de este para fines de investigación en psicología, así como se aclaró el derecho al resguardo de los datos, anonimato, confidencialidad, riesgos y beneficios.

\section{Instrumentos}

El instrumento contempló el consentimiento informado, preguntas de corte sociodemográfico y las escalas incluidas en el estudio de García-Castro y Pérez-Sánchez (2018), las cuales se detallan a continuación: 


\section{Escala de Miedo al Crimen}

Consiste en una adaptación de la escala del miedo al crimen por Ruíz (Ruíz, 2007; Ruíz y Turcios, 2009). Esta comprende cuatro ítems sobre el grado de temor al crimen que se siente en el hogar, el barrio, la ciudad y el país. Evalúa el miedo personal a la victimización. Cada reactivo tenía una escala Likert con cinco opciones que van desde el $1=$ nada, a 5= mucho. Presentó una confiabilidad promedio de $\alpha=0.75$, medida por el coeficiente alfa de Cronbach en el estudio de García-Castro y Pérez-Sánchez (2018).

\section{Escala de Transporte Narrativo}

Comprende una escala de 15 ítems, adaptada del estudio de García-Castro y Pérez-Sánchez (2018), en la cual las opciones van entre el 1 y el 5, significando el 1 "no se aplica en absoluto" y el 5 "se aplica mucho". Fue desarrollada originalmente por Green y Brock (2000). Mide el grado de implicación mental y emocional, así como la atención prestada a las noticias sobre delitos en los noticieros. Presenta elementos como "me imaginé en la escena de las noticias" y "presté toda mi atención a las noticias mientras las veía". En el estudio de García-Castro y Pérez- Sánchez (2018) se obtuvo un coeficiente alfa de Cronbach de $\alpha=0.76$.

\section{Procedimientos de recolección}

La recolección de datos se dio a través de medios virtuales, específicamente a través de las redes sociales Facebook y WhatsApp, donde se incluyó un enlace de Formulario de Google. Se empleó un muestreo no probabilístico por conveniencia utilizando el método de bola de nieve.

\section{Procedimientos de análisis}

Se realizaron análisis de confiabilidad de Cronbach, descriptivos, correlacionales y de frecuencias, utilizando el Programa Estadístico SPSS, versión 22 (SPSS Inc., Chicago, Illinois, USA).

\section{Resultados}

Se contó con una muestra de 157 sujetos, la cual estuvo compuesta por 87 mujeres $(55,4 \%)$ y 70 hombres $(44,6 \%)$ en edades entre los 18 y 67 años $(M=30,29 ; D E=10,88)$. De estos, 86 participantes eran de San José (54\%), 21 participantes de Heredia (13,4\%), 19 participantes de Cartago (12,1\%), 14 participantes de Alajuela (8,9\%), 14 participantes de Limón (8,9\%), 2 participantes de Guanacaste $(1,3 \%)$ y 1 participante de Puntarenas $(0,6 \%)$.

Además, un participante reportó un grado de escolaridad de primaria completa $(0,6 \%), 14$ participantes de secundaria incompleta $(8,9 \%), 24$ participantes de secundaria completa $(15,3 \%)$, 67 participantes de estudios superiores incompletos (42,7\%), 42 participantes de estudios superiores completos $(26,8 \%)$ y 9 participantes de estudios superiores de posgrado $(5,7 \%)$. 
Los participantes mostraron consumir de 0 a 112 horas semanales de noticias $(M=13,26$; $\mathrm{DE}=14,18)$; de los cuales 105 reportaron consumir más noticias a través de las redes sociales (66,9\%), 42 manifestaron consumir más noticias a través de la televisión (26,8\%), 8 reportaron consumir más noticias a través de radio $(5,1 \%)$ y 2 manifestaron consumir más noticias a través del periódico $(1,3 \%)$.

Los análisis de confiabilidad evidenciaron que la escala de Transporte Narrativo posee un nivel de confiabilidad aceptable $(\alpha=.78)$, mientras que la escala de Miedo al crimen reportó un excelente nivel de confiabilidad $(\alpha=.91)$.

Para la variable Transporte Narrativo, se reportaron puntuaciones de 1,47 a 4,87 (M=3,02; $\mathrm{DE}=0,67)$; mientras que para la variable Miedo, se obtuvieron puntuaciones de 1 a $5(\mathrm{M}=3,44$; $\mathrm{DE}=1,24)$, teniéndose en cuenta que estas escalas puntúan de 1 a 5 , donde a mayor valor, mayor transporte narrativo y miedo al crimen percibidos.

Al realizar el análisis de correlación simple, se halló una relación significativa y positiva entre el miedo al crimen y el transporte narrativo $(\mathrm{r}=.37 ; \mathrm{p}<.001)$, lo cual indica que, a mayor miedo, mayor transporte narrativo y viceversa. Además, se encontró una relación significativa y negativa del miedo al crimen con el estatus socioeconómico $(\mathrm{r}=-.23 ; \mathrm{p}<.001)$ y género $(\mathrm{r}=-.22$; $\mathrm{p}<.001$ ), lo que indica que, a mayor miedo, menor estatus socioeconómico, y viceversa; así como, que es el sexo femenino es el que percibe más miedo al crimen. En lo que respecta al transporte narrativo, se halló una relación significativa y positiva de esta variable con el consumo semanal de noticias $(\mathrm{r}=.25 ; \mathrm{p}<.001)$, de manera que, a mayor transporte narrativo, mayor consumo semanal de noticias, y viceversa (Tabla 1). 


\section{Tabla 1.}

Medias, desviaciones típicas y correlaciones simples entre las variables de estudio.

\begin{tabular}{lcccccccccc}
\hline \multicolumn{1}{c}{ Variable } & $a$ & $\mathrm{M}$ & $\mathrm{DE}$ & Miedo & Transporte & ESE & CSN & Sexo & Edad & GE \\
\cline { 2 - 9 } Miedo & .91 & 3.44 & 1.24 & - & $.37^{* *}$ & $-.23^{* *}$ & .06 & $-.22^{* *}$ & -.07 & -.07 \\
Transporte & .78 & 3.02 & .67 & & - & -.08 & $.25^{* *}$ & -.02 & -.03 & .02 \\
ESE & & 2.80 & 1.64 & & & - & .03 & .12 & $.26^{* *}$ & $.42^{* *}$ \\
CSN & & 13.26 & 14.18 & & & & - & .14 & -.08 & -.03 \\
Género & 1.45 & .50 & & & & & - & .10 & -.003 \\
Edad & 30.29 & 10.88 & & & & & & - & $.34^{* *}$ \\
GE & & 5.03 & 1.03 & & & & & & & -
\end{tabular}

Nota. ${ }^{*}$ p $<.001$, ESE: Estatus socioeconómico, CSN Consumo semanal de noticias, GE Grado de escolaridad

\section{Discusión}

Los resultados comprobaron que no existe una relación entre el miedo y el consumo semanal de noticias, lo cual ha sido encontrado también en el estudio de García- Castro y PérezSánchez (2018). Asimismo, no se demostró una relación entre la edad y la percepción del delito. En contraste a lo anterior, se halló que las mujeres y las personas de estrato social bajo perciben de forma más negativa el fenómeno de la delincuencia a partir del miedo al crimen, el cual a su vez se encuentra significativamente relacionado con el transporte narrativo (Fonseca y Sandoval, 2005; García-Castro y Pérez-Sánchez, 2018, Liebnitzky y Montero, 2013; Molina-Jácome, 2014).

El transporte narrativo, por su parte, es un buen indicador de percepción del delito, ya que su proceso se encuentra mediado por la atención, implicación y capacidad cognitiva (García-Castro y Pérez-Sánchez, 2018), las cuales influyen en la percepción, considerada una variable subjetiva basada en la percepción objetiva, donde también interviene lo afectivo de manera determinante (Villalba, 2017).

De ahí que la exposición frecuente a noticias en torno al crimen influya en la manera en que las personas se sienten y perciben el fenómeno de la delincuencia, generando principalmente sentimientos de preocupación en el ámbito afectivo, así como miedo si se trata de noticias locales (García-Castro y Pérez-Sánchez, 2018; Soto, 2005). 
A su vez, las noticias de carácter delictivo tienen la capacidad de incidir en las cogniciones de los sujetos y crear aprendizajes significativos, esto a través de los medios de comunicación como importantes agentes socializadores, lo cual incide en la victimización directa e indirecta y hace del Síndrome del mundo cruel una epidemia (Gerbner et al., 1986; Igartua y Gerbner, 2002; Molina-Jácome, 2014; Morgan et al., 2014; Villalba, 2017).

Además, se ha establecido que en gran medida las noticias se encuentran determinadas a partir de la agenda pública y, sobre todo, de la política, por lo que los medios de comunicación acaban desempeñando un rol de control social con base en diversos intereses, influyendo en la audiencia y a su vez generando en esta una valoración positiva o negativa de las temáticas transmitidas y otras íntimamente relacionadas, puesto que, vía enlace asociativo, es posible activar pensamientos de significado semejante a partir de conceptos afines semánticamente (Freidenberg et al., 1999; Muñiz et al., 2015).

El miedo como factor de la delincuencia ha sido ampliamente estudiado a través de una multiplicidad de factores, tanto a nivel micro, considerando aspectos individuales como sexo, edad, escolaridad, estatus laboral, tipo de ocupación u otras; así como a nivel macro a través de la marginación en los barrios, la confianza en la policía local y el contenido de los noticieros (GarcíaCastro y Pérez-Sánchez, 2018; Molina-Jácome, 2014).

Varios estudios han considerado que los individuos de estrato social bajo son los que poseen mayores niveles de miedo al crimen (Fonseca y Sandoval, 2005; García-Castro y PérezSánchez, 2018, Liebnitzky y Montero, 2013; Molina-Jácome, 2014), un aspecto que también ha sido apoyado por la presente investigación. Esto puede evidenciarse desde una perspectiva de vulnerabilidad; por lo tanto, es probable que personas de estrato social bajo convivan en lugares marginados donde existe una probabilidad mayor de ser víctima de un crimen.

Lo anterior resulta a su vez un indicio para hacer constar que la segunda hipótesis "(H 2) el sexo femenino tiene una percepción de la delincuencia más negativa que el sexo masculino" fue apoyada. Se ha asociado que las percepciones de vulnerabilidad de las mujeres consigo mismas influyen en un mayor miedo al crimen, lo cual repercute en que estas exhiban una percepción negativa de la delincuencia, evitando lugares públicos o buscando significativos físicamente fuertes que ejerzan un rol protector para con ellas (García-Castro y Pérez-Sánchez, 2018; Ruíz, 2007).

En lo que respecta a la tercera hipótesis " $(\mathrm{H} 3)$ el medio de comunicación por el que se consumen más noticias delictivas son las redes sociales" fue apoyada. Una explicación tentativa para esto es que existe una tendencia a que los individuos de estrato social bajo se informen a través de la televisión (Fonseca y Sandoval, 2005), mientras que se ha evidenciado que las personas de estrato social medio y alto son las que tienen más acceso a las redes sociales, principalmente el grupo etario comprendido entre los 21 a 30 años (El Salvador S.A de C.V, 2017); de ahí que es probable que opten por este medio para informarse.

Finalmente, no se encontró apoyo para la cuarta hipótesis " $(\mathrm{H} 4)$ a mayor edad, mayor percepción negativa de la delincuencia". Esto podría deberse a que se contó con una muestra significativa de participantes jóvenes, por lo que no fue posible trazar resultados concluyentes al respecto. 
Cabe mencionar que hasta ahora las investigaciones se han interesado en estudiar el miedo al crimen y la percepción de la delincuencia mayoritariamente a partir de la televisión, pues se ha comprobado que tiene un gran impacto en las percepciones de riesgo y miedo al crimen, esto a través de la interpretación de imágenes e información (Callanan, 2012; Koziner, 2013); no obstante, los medios de comunicación en general están dotados de una gran suma de canales semánticos, de ahí que podría existir una mayor percepción negativa de la delincuencia tras la exposición al consumo de noticias delictivas desde diversos medios de comunicación, considerando especialmente las redes sociales, las cuales han tomado auge tras el vertiginoso desarrollo de la tecnología, y que además comparten características similares con la televisión.

\section{Referencias}

Álvarez-Gálvez, J. (2012). Modelos teóricos sobre los efectos de los medios de comunicación de masas. Documento de trabajo, 2. https://eprints.ucm.es/45089/

Browne, M., y Tomicic, V. (2011). Crimen y temor: el rol de los medios / Crime and Fear: The Role of the Media. Cuadernos.Info, (20), 21-36. https://doi.org/10.7764/cdi.20.110

Callanan, V. J. (2012). Media Consumption, Perceptions of Crime Risk and Fear of Crime: Examining Race/Ethnic Differences. Sociological Perspectives, 55(1), 93- 115. https://doi.org/10.1525/sop.2012.55.1.93

Carmona, M. S. (2002). Los medios de comunicación masiva en Nuevo León (Tesis de maestría) Universidad de Nuevo León. http://eprints.uanl.mx/974/1/1020148231.PDF

El Salvador S.A. de C.V., E. \& Y. (2017). Comportamiento en el uso de redes sociales en Centroamérica y el Caribe. Realidad Empresarial, (3), 14-19. https://doi.org/10.5377/reuca.v0i3.5962

Focás, B. (2018). Miedo al crimen, prevención del delito y narcotráfico: desafíos para las políticas públicas de seguridad ciudadana en América Latina. Entrevista a Lucía Dammert. Urvio. Revista Latinoamericana de Seguridad Ciudadana, (5), 102-108. https://doi.org/10.17141/urvio.22.2018.3183

Fonseca, K., y Sandoval, C. (2005). Medios de comunicación e (in)seguridad ciudadana en Costa Rica. San José, Costa Rica: Programa de las Naciones Unidas para el Desarrollo. https://www.kerwa.ucr.ac.cr/handle/10669/79438

Freidenberg, F., D'Adamo, O., y García, V. (1999). Medios de comunicación y política. Un estudio del efecto de priming durante una campaña electoral. Entrecaminos Washington: Georgetown University Center for Latin American Studies, 4, 133-150. http://www.copmadrid.es/webcopm/recursos/pol7.pdf

García-Castro, J. D. (2012). Percepción de criminalidad en Costa Rica: clases sociales, televisión e ideología. Becas de Investigación (Serie Documentos de Trabajo). Buenos Aires: CLACSO. http://biblioteca.clacso.edu.ar/clacso/becas/20120509124519/garciacastro.pdf 
García-Castro, J. D., \& Pérez-Sánchez, R. (2018). Fear of Crime and Cultivation Effect. Social and Psychological Predictors. Universitas Psychologica, 17(3), 1-14. https://doi.org/10.11144/Javeriana.upsy17-3.fcce

García-Castro, J. D., y Barrantes-Umaña, B. (2016). Percepción y miedo al crimen: Revisión de artículos en español 2004-2014. Revista Reflexiones, 95(1), 87-100. https://doi.org/10.15517/rr.v95i1.28201

Gerbner, G., Gross, L., Morgan, M., \& Signorielli, N. (1986). Living with television: The dynamics of the cultivation process. En J. Bryant \& D. Zillman (eds.). Perspectives on media effects (pp. 17-40). Hilldale, N.J: Lawrence Erlbaum Associates.

Green, M. C. (2004). Transportation Into Narrative Worlds: The Role of Prior Knowledge and Perceived Realism. Discourse Processes, 38(2), 247-266. https://doi.org/10.1207/s15326950dp3802 5

Green, M., \& Brock, T. (2000). The role of transportation in the persuasiveness of public narratives. Journal of Personality and Social Psychology, 79(5), 701-721. https://doi.org/10.1037/0022-3514.79.5.701

Huhn, S. (2012). Criminalidad y discurso en Costa Rica: reflexiones críticas sobre un problema social. San José, Costa Rica: FLACSO. http://rosalux.org.mx/docs/CriminalidadCR.pdf

Igartua, J. J., y Gerbner, G. (2002). Violencia y televisión: nuestro medio ambiente cultural. Entrevista con George Gerbner. Cultura y Educación, 14(1), 55-61. https://doi.org/10.1174/113564002317348110

Jaume, L., y Etchezahar, E. (2013). La justificación del sistema: diferencias teórico conceptuales entre la perspectiva sociológica y psicológica. $X$ Jornadas de Sociología. http://cdsa.aacademica.org/000-038/326

Jost, J. T., Banaji, M., \& Nosek, B. (2004). A Decade of System Justification Theory: Accumulated Evidence of Conscious and Unconscious Bolstering of the Status Quo. Political Psychology, 25(6), 881-919. https://doi.org/10.1111/j.1467-9221.2004.00402.x

Jost, J. T., \& Hunyady, O. (2005). Antecedents and consequences of system-justifying ideologies. Current Directions in Psychological Science, 14(5), 260-265. https://doi.org/10.1111/j.0963-7214.2005.00377.x

Jost, J. T., Ledgerwood, A., \& Hardin, C. D. (2008). Shared reality, system justification, and the relational basis of ideological beliefs. Social and Personality Psychology Compass, 2(1), 171-186. https://doi.org/10.1111/j.1751-9004.2007.00056.x

Koziner, N. S. (2013). Antecedentes y fundamentos de la teoría del framing en comunicación. Austral Comunicación, $\quad 2(1), \quad 1-25$. http://www.austral.edu.ar/ojs/index.php/australcomunicacion/article/view/53

Liebnitzky, J., \& Montero, M. (2013). Miedo al crimen en estudiantes de la ciudad de Caracas. Psicologia \& Sociedade, 25(1),

152-162. http://submission.scielo.br/index.php/psoc/article/view/71588/8148 
Molina-Jácome, I. (2014). Miedo al crimen y medios de comunicación: una revisión de la Revista

Criminalidad, $56(3)$

9-23. http://www.scielo.org.co/scielo.php?script=sci abstract\&pid=S1794$31082014000300002 \& \operatorname{lng}=\mathrm{es} \& \mathrm{nrm}=\mathrm{is}$

Morgan, M., Shanahan, J., \& Signorielli, N. (2014). Cultivation Theory in the Twenty- First Century. En Fortner, R. S. \& Fackler, P. M. (Eds), The Handbook of Media and Mass Communication Theory, 480- 497. John Wiley and Sons https://doi.org/10.1002/9781118591178.ch26

Muñiz, V., Fonseca, R. A., y Castillo, Y. (2015). Estudios sobre Agenda Setting en Cuba (20102015). Tendencias generales de investigación en pregrado. Razón y Palabra, 19(92), 1-29. http://www.redalyc.org/articulo.oa? $\mathrm{id}=199543036007$

Ruiz, J. I. (2007). Cultura ciudadana, miedo al crimen y victimización: un análisis de sus interrelaciones desde la perspectiva del tejido social. Acta Colombiana de Psicología, 10(1), 65-74. http://www.redalyc.org/articulo.oa?id=79810107

Ruiz, J. I, \& Turcios, L. A. (2009). Percepción de seguridad, victimización y cultura ciudadana: sus relaciones en cinco contextos iberoamericanos. Pensamiento Psicológico, 6(13), 193202. http://www.redalyc.org/articulo.oa?id=80112469013

Sáenz, M. A. (2003). El papel de los medios de comunicación en la construcción social del fenómeno de la criminalidad. Cuadernos de estudio del Ministerio Público de Costa Rica $N^{\circ}$. Revista Especializada en Criminología y Derecho Penal, (8), 49-58. https://ministeriopublico.poder-judicial.go.cr/documentos/biblioteca digital/cuadernosde-estudio/cuaderno\%20N8.pdf

Soto, S. (2005). La influencia de los medios en la percepción social de la delincuencia. Revista Electrónica de Ciencia Penal y Criminología, (7), 1-46. https://dialnet.unirioja.es/servlet/articulo?codigo=1215815

Téllez, W. J. (2015). Explorando la percepción de la delincuencia como principal problema en América Latina. Revista Internacional de Investigación en Ciencias Sociales, 11(2), 195208. https://dialnet.unirioja.es/servlet/articulo?codigo=5297967

Van Laer, T., De Ruyter, K., Visconti, L. M., \& Wetzels, M. (2014). The Extended TransportationImagery Model: A Meta-Analysis of the Antecedents and Consequences of Consumers' Narrative Transportation. Journal of Consumer Research, 40(5), 797-817. https://doi.org/10.1086/673383

Vergara, A. (2016). El discurso sobre la criminalidad en las noticias televisivas en Costa Rica. Logos: Revista de Lingüística, Filosofía y Literatura, 26(1), 241-259. https://doi.org/10.15443/RL26019

Villalba, J. (2017). Variables predictivas del miedo al delito a nivel individual (Tesis de doctorado). Universidad Nacional de Educación a Distancia]. http://espacio.uned.es/fez/view/tesisuned:ED-Pg-DeryCSoc-Jvillalba 